\title{
Worldwide Esophageal Cancer Collaboration: pathologic staging data
}

T. W. Rice, ${ }^{1}$ L.-Q. Chen, ${ }^{2}$ W. L. Hofstetter, ${ }^{3}$ B.M. Smithers, ${ }^{4}$ V. W. Rusch,${ }^{5}$ B. P. L. Wijnhoven, ${ }^{6}$ K. L. Chen, ${ }^{7}$ A. R. Davies, ${ }^{8}$ X. B. D’Journo, ${ }^{9}$ K. A. Kesler, ${ }^{10}$ J. D. Luketich, ${ }^{11}$ M. K. Ferguson, ${ }^{12}$ J. V. Räsänen, ${ }^{13}$ R. van Hillegersberg, ${ }^{14}$ W. Fang, ${ }^{15}$ L. Durand,${ }^{16}$ I. Cecconello, ${ }^{17}$ W. H. Allum,${ }^{18}$ R. J. Cerfolio, ${ }^{19}$ M. Pera,${ }^{20}$ S. M. Griffin, ${ }^{21}$ R. Burger,${ }^{22}$ J.-F. Liu,${ }^{23}$ M. S. Allen, ${ }^{24}$ S. Law,${ }^{25}$ T. J. Watson, ${ }^{26}$ G. E. Darling, ${ }^{27}$ W. J. Scott,${ }^{28}$ A. Duranceau, ${ }^{29}$ C. E. Denlinger, ${ }^{30}$ P. H. Schipper, ${ }^{31}$ T. E. M. R. Lerut ${ }^{32}$ M. B. Orringer, ${ }^{33}$ H. Ishwaran, ${ }^{34}$ C. Apperson-Hansen, ${ }^{35}$ L. M. DiPaola, ${ }^{1}$ M. E. Semple, ${ }^{1}$ E. H. Blackstone ${ }^{1}$

${ }^{1}$ Cleveland Clinic, Cleveland, Ohio, USA, ${ }^{2}$ West China Hospital of Sichuan University, Chengdu, Sichuan, China,

${ }^{3}$ University of Texas MD Anderson Hospital, Houston, Texas, USA, ${ }^{4}$ University of Queensland, Princess Alexandra Hospital, Brisbane, Australia, ${ }^{5}$ Memorial Sloan-Kettering Cancer Center, New York, New York, USA, ${ }^{6}$ Erasmus Medical Center, Rotterdam, The Netherlands, ${ }^{7}$ Beijing Cancer Hospital, Beijing, China, ${ }^{8}$ Guy's \& St Thomas' Hospitals, London, England, ${ }^{9}$ Hôpital Nord, Marseille, France, ${ }^{10}$ Indiana University Medical Center, Indianapolis, Indiana, USA, ${ }^{11}$ University of Pittsburgh Medical Center, Pittsburgh, Pennsylvania, USA, ${ }^{12}$ Department of Surgery, The University of Chicago, Chicago, Illinois, USA, ${ }^{13}$ Helsinki University Hospital, Helsinki, Finland, ${ }^{14}$ University Medical Center Utrecht, Utrecht, The Netherlands, ${ }^{15}$ Shanghai Chest Hospital, Shanghai, China, ${ }^{16}$ Hospital de Clinicas, University of Buenos Aires, Buenos Aires, Argentina, ${ }^{17}$ University of São Paulo School of Medicine, São Paulo, Brazil, ${ }^{18}$ Royal Marsden NHS Foundation Trust, London, UK, ${ }^{19}$ Section of Thoracic Surgery, University of Alabama at Birmingham, Birmingham, Alabama, USA, ${ }^{20}$ Hospital Universitario del Mar, Barcelona, Spain, ${ }^{21}$ University of Newcastle upon Tyne, Newcastle, United Kingdom, ${ }^{22}$ University of Cape Town, Groote Schuur Hospital, Cape Town, South Africa, ${ }^{23}$ Fourth Hospital of Hebei Medical University, Shijiazhuang, Hebei, China, ${ }^{24}$ Mayo Clinic, Rochester, Minnesota, USA, ${ }^{25}$ University of Hong Kong Medical Center, Queen Mary Hospital, Hong Kong, China ${ }^{26}$ University of Rochester, Rochester, New York, USA,

${ }^{27}$ Toronto General Hospital, Toronto, Ontario, Canada, ${ }^{28}$ Fox Chase Cancer Center, Philadelphia, Pennsylvania, USA, ${ }^{29}$ University of Montreal, Montreal, Quebec, Canada, ${ }^{30}$ Medical University of South Carolina, Charleston, South Carolina, USA, ${ }^{31}$ Oregon Health \& Science University, Portland, Oregon, USA, ${ }^{32}$ University Ziekenhuizen Leuven, Leuven, Belgium, ${ }^{33}$ University of Michigan, Ann Arbor, Michigan, USA, ${ }^{34}$ University of Miami, Miami, Florida, USA, and ${ }^{35}$ Case Western Reserve University, Cleveland, Ohio, USA

SUMMARY. We report data-simple descriptions of patient characteristics, cancer categories, and non-riskadjusted survival-for patients with pathologically staged cancer of the esophagus and esophagogastric junction after resection or ablation with no preoperative therapy from the Worldwide Esophageal Cancer Collaboration (WECC). Thirty-three institutions from six continents submitted de-identified data using standard definitions: demographics, comorbidities, clinical cancer categories, and all-cause mortality from first management decision. Of 13,300 patients, 5,631 had squamous cell carcinoma, 7,558 adenocarcinoma, 85 adenosquamous carcinoma, and 26 undifferentiated carcinoma. Patients were older $(62$ years) men $(80 \%)$ with normal body mass index $(51 \%)$, little weight loss $(1.8 \mathrm{~kg}), 0-2$ ECOG performance status $(83 \%)$, and a history of smoking $(70 \%)$. Cancers were pT1 (24\%), pT2 (15\%), pT3 (50\%), pN0 (52\%), pM0 (93\%), and pG2-G3 (78\%); most involved distal esophagus (71\%). Non-risk-adjusted survival for both squamous cell carcinoma and adenocarcinoma was monotonic and distinctive across pTNM. Survival was more distinctive for adenocarcinoma than squamous cell carcinoma when $\mathrm{pT}$ was ordered by $\mathrm{pN}$. Survival for pTis-1 adenocarcinoma was better than for squamous cell carcinoma, although monotonic and distinctive for both. WECC pathologic staging data is improved over that of the 7th edition, with more patients studied and patient and cancer variables collected. These data will be the basis for the 8th

Address correspondence to: Thomas W. Rice, MD, Department of Thoracic and Cardiovascular Surgery, Cleveland Clinic; 9500 Euclid Avenue/Desk JJ-40; Cleveland, OH 44195, USA. Email: ricet@ccf.org 
edition cancer staging manuals following risk adjustment for patient, cancer, and treatment characteristics, and should direct 9th edition data collection. However, the role of pure pathologic staging as the principal point of reference for esophageal cancer staging is waning.

$K E Y$ WORDS: survival, cancer staging, esophagectomy, decision-making, prognostication.

\section{INTRODUCTION}

Staging of cancer of the esophagus and esophagogastric junction in the 7th edition AJCC and UICC cancer staging manuals ${ }^{1,2}$ was based on pathologic stage after esophagectomy alone, derived from the Worldwide Esophageal Cancer Collaboration (WECC) data. ${ }^{3}$ Unanswered questions from the previous WECC effort were (1) Do squamous cell carcinoma and adenocarcinoma share stage grouping for advanced cancers? (2) Can early-stage cancers be subgrouped? (3) What is the effect of postoperative adjuvant therapy? (4) What is the effect of endoscopic therapies and minimally invasive techniques? Answering these questions requires a larger, more comprehensive pathologic data set of patient characteristics and pathologic cancer categories. Therefore, a sixcontinent worldwide WECC effort was mounted to (1) answer these questions, (2) facilitate postresection prognostication, (3) improve post-resection decisionmaking, and 4) prepare for the 8th edition cancer staging manuals.

In this article, we report the descriptive data set of patient characteristics and cancer categories of individuals with pathologically staged esophageal cancer after resection or ablative therapy and non-risk-adjusted analysis of survival that begins to address these aims.

\section{PATIENTS AND METHODS}

Data

In 2012, institutions worldwide were invited to participate in WECC, aimed at constructing refined datadriven esophageal cancer staging for the 8th edition of the cancer staging manuals. ${ }^{4}$ Data were requested in completely deidentified form (Health Insurance Portability and Accountability Act research standards) for analysis, using a set of required variables with standard definitions. Local ethics-board approval of the databases and data-use agreements was executed with Cleveland Clinic. Variables included demographics, comorbidities, cancer categories, cancer treatment, and time-related outcomes. The Case Cancer Institutional Review Board of Case Western Reserve University and Cleveland Clinic Institutional Review Board approved the entire project. This paper reports results of clinical data from 33 institutions whose data were (C) 2016 International Society for Diseases of the Esophagus submitted by September 30, 2014, and were cleaned and adjudicated (Appendix).

\section{Patients}

At these institutions, 13,300 patients with epithelial cancer of the esophagus or esophagogastric junction had pathologic staging after esophagectomy or endoscopic resection or ablation alone. The majority of patients were older men with normal $(51 \%)$ or overweight $(29 \%)$ body mass index, no weight loss, and 0-2 Eastern Cooperative Oncology Group (ECOG) performance status (Table 1 and Supporting Information Table S1). Comorbidities were present in a minority of patients, with cardiopulmonary comorbidities predominating. These data revealed that patients with pure adenocarcinoma were older, far less likely to be female, considerably larger, more likely to have ECOG performance status $0-1$, more likely to have comorbidities, including diabetes, coronary artery disease, arrhythmia, hypertension, and peripheral artery disease, and more likely to have lower forced expiratory volume in 1 second (FEV1) and forced vital capacity (FVC) than those with pure squamous cell carcinoma (Table 1 and Supporting Information Table S1). Although six continents are represented, most patients in the data set were treated in North America, Europe, and Asia. Patients with adenocarcinoma lived predominantly in the West and those with squamous cell carcinoma in the East.

\section{Treatment}

Most patients had an esophagectomy (Table 2 and Supporting Information Table S2), some performed less invasively. A small number of patients had endoscopic therapy. In both histopathologic cell types, more than $70 \%$ of patients had more than 11 regional lymph nodes resected, and 30\% to $40 \%$ had more than 20 resected. Postoperative adjuvant therapy was used in twice as many squamous cell carcinoma patients as in adenocarcinoma patients.

\section{Endpoint}

The endpoint was all-cause mortality from first management decision. Median potential follow-up, ${ }^{5}$ if there were no deaths, was 9.8 years $(25 \%>15$ years, $10 \%>21$ years), but considering deaths in this elderly population with a rapidly lethal cancer, overall median 
Table 1 Characteristics of patients receiving resection or ablation without preoperative therapy for pure squamous cell carcinoma and pure adenocarcinoma of the esophagus

\begin{tabular}{|c|c|c|c|c|}
\hline \multirow[b]{2}{*}{ Characteristic } & \multicolumn{2}{|c|}{ Squamous Cell Cancer $(n=5,631)$} & \multicolumn{2}{|c|}{ Adenocarcinoma $(n=7,558)$} \\
\hline & $n^{\dagger}$ & $\begin{array}{l}\text { No. }(\%) \text { or } \\
\text { Mean } \pm \text { SD }\end{array}$ & $n^{\dagger}$ & $\begin{array}{l}\text { No. }(\%) \text { or } \\
\text { Mean } \pm \text { SD }\end{array}$ \\
\hline \multicolumn{5}{|l|}{ Demographics } \\
\hline Age (years) & 5,597 & $60 \pm 9.7$ & 7,290 & $64 \pm 11$ \\
\hline Female & 5,631 & $1,559(28)$ & 7,556 & $1,120(15)$ \\
\hline Body mass index $\left(\mathrm{kg} / \mathrm{m}^{2}\right)$ & 3,318 & $22 \pm 3.4$ & 3,978 & $27 \pm 5.0$ \\
\hline Weight loss $(\mathrm{kg})$ & 3,469 & $1.5 \pm 4.4$ & 4,168 & $2.2 \pm 10$ \\
\hline \multicolumn{5}{|l|}{ Comorbidities } \\
\hline ECOG performance status & 2,477 & & 1,262 & \\
\hline 0 & & 427 (17) & & $486(39)$ \\
\hline 1 & & $285(12)$ & & $576(46)$ \\
\hline 2 & & $1,233(50)$ & & $123(9.7)$ \\
\hline 3 & & $526(21)$ & & $68(5.4)$ \\
\hline 4 & & $6(0.24)$ & & $9(0.71)$ \\
\hline Diabetes & 5,158 & $190(3.7)$ & 5,945 & $717(12)$ \\
\hline IDDM & 5,120 & $35(0.68)$ & 5,681 & $112(2.0)$ \\
\hline NIDDM & 5,120 & $117(2.3)$ & 5,681 & $341(6.0)$ \\
\hline Coronary artery disease & 2,990 & $113(3.8)$ & 2,387 & $459(19)$ \\
\hline Arrhythmia & 2,838 & $39(1.4)$ & 1,775 & $64(3.6)$ \\
\hline Hypertension & 3,943 & $640(16)$ & 4,955 & $1,491(30)$ \\
\hline Peripheral arterial disease & 3,375 & $56(1.7)$ & 3,202 & $145(4.5)$ \\
\hline Smoker & 3,668 & $2,580(70)$ & 5,106 & $3,523(69)$ \\
\hline Past & 3,281 & $986(30)$ & 3,999 & $1,434(36)$ \\
\hline Current & 3,281 & $1,207(37)$ & 3,999 & $982(25)$ \\
\hline FEV1 $(\%)$ & 2,871 & $98 \pm 21$ & 3,053 & $95 \pm 20$ \\
\hline $\mathrm{FVC}(\%)$ & 2,763 & $110 \pm 21$ & 2,489 & $103 \pm 19$ \\
\hline Creatinine (mg/dL) & 2,314 & $76 \pm 16$ & 755 & $73 \pm 34$ \\
\hline Bilirubin (mg/dL) & 2,274 & $13 \pm 6.3$ & 580 & $11 \pm 6.2$ \\
\hline Decade of treatment & 5,630 & & 7,558 & \\
\hline $1970-1979$ & & $67(1.2)$ & & $39(0.52)$ \\
\hline $1980-1989$ & & $1,092(19)$ & & $288(3.8)$ \\
\hline 1990-1999 & & $888(16)$ & & $2,518(33)$ \\
\hline $2000-2010$ & & $1,964(35)$ & & $3,992(53)$ \\
\hline $2010-2013$ & & $1,619(29)$ & & $721(9.5)$ \\
\hline Continent & 5,631 & & 7,558 & \\
\hline North America & & 964 (17) & & $3,934(52)$ \\
\hline Europe & & $904(16)$ & & $2,774(37)$ \\
\hline Asia & & $3,633(65)$ & & $309(4.1)$ \\
\hline Australia & & $79(1.4)$ & & $358(4.7)$ \\
\hline South America & & $38(0.67)$ & & $182(2.4)$ \\
\hline Africa & & $13(0.23)$ & & $1(0.013)$ \\
\hline
\end{tabular}

Patient characteristics of those with adenosquamous and undifferentiated carcinoma are shown in Supporting Information Table S1. $†$ Patients with data available. ECOG, Eastern Cooperative Oncology Group; FEV1 (\%), forced expiratory volume in 1 second (percent of predicted); FVC (\%), forced vital capacity (percent of predicted); IDDM, insulin-dependent diabetes mellitus; NIDDM, non-insulindependent diabetes mellitus; SD, standard deviation.

follow-up was 1.6 years; median follow-up for surviving patients was 3.0 years, with $25 \%$ followed more than 5.9 years and $10 \%$ more than 9.1 years.

\section{Data analysis}

For analysis, patients with adenosquamous and undifferentiated carcinoma (Supporting Information Tables $\mathrm{S} 1$ and S2) were considered in both squamous cell carcinoma and adenocarcinoma data sets. Survival was estimated by the Kaplan-Meier method, and these estimates are accompanied by $68 \%$ confidence limits, equivalent to \pm 1 standard error. Survival has been simply stratified by a number of patient characteristics and cancer categories, with no risk adjustment. The hazard function for death was estimated by a parametric temporal decomposition method (for additional details, see http://www.lerner.ccf.org/qhs/software/ hazard). ${ }^{6}$ Continuous variables are summarized by mean \pm standard deviation and categorical variables by frequency and percentage.

\section{RESULTS}

\section{Pathologic cancer categories}

Histopathologic cell type was squamous cell carcinoma in 5,631, adenocarcinoma in 7,558, adenosquamous carcinoma in 85 , and undifferentiated carcinoma in 26 . The majority of cancers invaded outside the esophageal wall (pT3-4a) (Table 3 and Supporting Information Table S3). Because 31\% of adenocarcinoma patients had pT1 cancers, there was a near equal chance of invasion confined to the esophageal wall (pTis-T2) as invasion outside the esophageal wall (pT3-4a); the majority of squamous 
Table 2 Treatment received by patients for pure squamous cell carcinoma and pure adenocarcinoma of the esophagus

\begin{tabular}{lcc}
\hline & $\begin{array}{c}\text { Squamous } \\
\text { cell cancer } \\
(n=5,631)\end{array}$ & $\begin{array}{c}\text { Adenocarcinoma } \\
(n=7,558) \\
\text { No. }(\%)\end{array}$ \\
Characteristic & No. $\%)$ & \\
\hline Resection & & $7,526(100)$ \\
Esophagectomy & $5,625(100)$ & $399(5.3)$ \\
Less invasive & $92(1.6)$ & $32(0.42)$ \\
Endoscopic & $6(0.11)$ & $124(4)$ \\
Lymph nodes resected & $133(4)$ & $218(7.1)$ \\
0 & $252(7.7)$ & $413(13)$ \\
$1-5$ & $509(15)$ & $1,043(34)$ \\
$6-10$ & $1,388(42)$ & $626(20)$ \\
$11-20$ & $649(20)$ & $647(21)$ \\
$21-30$ & $354(11)$ & 4,487 \\
-30 & 2,346 & \\
Unknown & & $6,792(90)$ \\
Resection margin & $5,241(93)$ & $596(7.9)$ \\
R0 & $316(5.6)$ & $170(2.2)$ \\
R1 & $74(1.3)$ & \\
R2 & & $406(5.4)$ \\
Adjuvant therapy & $413(7.3)$ & $403(5.3)$ \\
Chemoradiotherapy & $646(11)$ & $128(1.7)$ \\
Chemotherapy & $253(4.5)$ & \\
Radiotherapy &
\end{tabular}

Treatment received by patients with adenosquamous and undifferentiated carcinoma is shown in Supporting Information Table S2. $\dagger$ Only total number of positive nodes was reported in 2,193 squamous cell carcinoma cases and 3,884 adenocarcinoma cases, so only the minimum number was known (a so-called "floor" effect).

cell carcinomas invaded outside the esophageal wall (Table 3 and Supporting Information Table S3). A nearly equal number of cancers were free of regional lymph node metastases ( $\mathrm{pN} 0$ ) as had metastases $(\mathrm{pN}+)$ for both histopathologic cell types. Frequency of number of positive lymph nodes progressively decreased for both histopathologic cell types, with $4.1 \%$ of squamous cell carcinomas and $16 \%$ of adenocarcinomas having 7 or more positive lymph nodes. Extracapsular lymph node invasion (ECLNI) and lymphovascular invasion (LVI), infrequently recorded, were nearly three times more likely in pure adenocarcinoma compared with squamous cell carcinoma. Few cancers had distant metastases (pM). Most cancers were G2/G3. Adenocarcinomas were located predominantly in the lower esophagus and squamous cell carcinoma in the middle and lower esophagus.

\section{Non-risk-adjusted survival}

Overall survival was $98 \%, 77 \%, 39 \%$, and $27 \%$ at 30 days and 1, 5, and 10 years (Supporting Information Fig. S1). Instantaneous early risk of death peaked differently between cell types: within months of first management decision for adenocarcinoma and within a year for squamous cell carcinoma. It then decreased to a constant rate of $7 \%$ per year after 5 years for both cell types (Supporting Information Fig. S2).

(C) 2016 International Society for Diseases of the Esophagus

\section{Pathologic cancer categories (pTNM)}

Survival decreased monotonically and distinctively with increasing depth of tumor invasion (pT; Fig. 1), pT1 subsets (Supporting Information Fig. S3), presence of regional lymph node metastases ( $\mathrm{pN}$; Fig. 2), and increasing number of cancer-positive regional lymph nodes (Supporting Information Fig. S4). For pN0 cancers, survival decreased distinctively and monotonically with respect to $\mathrm{pT}$ categories for both histopathologic cell types (Fig. 3). Survival for $\mathrm{pN}+$ cancers was poor (Fig. 4). It was monotonic by pT category for both histopathologic cell types, but less distinctive by $\mathrm{pT}$ for

Table 3 Pathologic cancer categories of patients receiving resection or ablation without preoperative therapy for pure squamous cell carcinoma and pure adenocarcinoma of the esophagus

\begin{tabular}{|c|c|c|}
\hline Category & $\begin{array}{c}\text { Squamous cell } \\
\text { cancer } \\
(n=5,631) \\
\text { No. }(\%)\end{array}$ & $\begin{array}{c}\text { Adenocarcinoma } \\
(n=7,558) \\
\text { No. }(\%)\end{array}$ \\
\hline \multicolumn{3}{|l|}{$p T$} \\
\hline pT0 & $0(0)$ & $0(0)$ \\
\hline pTis & $99(1.8)$ & $410(5.4)$ \\
\hline pT1 & $867(15)$ & $2,326(31)$ \\
\hline pT2 & 1,047 (19) & $947(13)$ \\
\hline pT3 & 3,024 (54) & $3,604(48)$ \\
\hline pT4a & $592(11)$ & $245(3.3)$ \\
\hline pTX & 2 & 26 \\
\hline \multicolumn{3}{|l|}{$p N$} \\
\hline $\mathrm{pN} 0$ & $3,198(57)$ & $3,666(48)$ \\
\hline $\mathrm{pN}+$ & $2,425(43)$ & $3,892(52)$ \\
\hline $\mathrm{pN} 1$ & $1,337(58)^{\mathrm{a}}$ & $1,159(34)^{\mathrm{b}}$ \\
\hline $\mathrm{pN} 2$ & $722(32)^{\mathrm{a}}$ & $1,130(34)^{b}$ \\
\hline $\mathrm{pN} 3$ & $227(10)^{\mathrm{a}}$ & $1,080(32)^{\mathrm{b}}$ \\
\hline $\mathrm{pNX}$ & 8 & 48 \\
\hline \multicolumn{3}{|l|}{ Number of positive nodes } \\
\hline 0 & $3,192(58)$ & $3,586(52)$ \\
\hline 1 & $849(15)$ & $679(9.8)$ \\
\hline 2 & 488 (8.9) & $480(6.9)$ \\
\hline 3 & $293(5.3)$ & $369(5.3)$ \\
\hline 4 & $221(4.0)$ & $284(4.1)$ \\
\hline 5 & $115(2.1)$ & $272(3.9)$ \\
\hline 6 & $93(1.7)$ & 205 (2.9) \\
\hline 7 or more & $227(4.1)$ & $1,080(16)$ \\
\hline Unknown & 153 & 603 \\
\hline ECLNI & $97(17)^{\mathrm{c}}$ & $396(47)^{\mathrm{d}}$ \\
\hline Lymphovascular invasion & $492(16)^{\mathrm{e}}$ & $1,005(44)^{\mathrm{f}}$ \\
\hline \multicolumn{3}{|l|}{$p M$} \\
\hline $\mathrm{pM} 0$ & $5,404(96)$ & $6,908(91)$ \\
\hline pM1 & $220(3.9)$ & $650(8.6)$ \\
\hline \multicolumn{3}{|l|}{ Grade $^{\mathrm{g}}$} \\
\hline pG1 & $1,233(24)$ & $1,216(20)$ \\
\hline pG2 & 1,973 (39) & $1,974(33)$ \\
\hline pG3 & $1,915(37)$ & $2,851(47)$ \\
\hline pGX & 507 & 1,517 \\
\hline \multicolumn{3}{|l|}{ Location } \\
\hline pUpper & $574(11)$ & $55(0.86)$ \\
\hline pMiddle & 2,542 (49) & $253(3.9)$ \\
\hline pLower & $2,083(40)$ & $6,114(95)$ \\
\hline pLocationX & 432 & 1,136 \\
\hline
\end{tabular}

Pathologic cancer categories of those with adenosquamous and undifferentiated carcinoma are shown in Supporting Information Table S3. a. Data available for 2,286 patients. b. Data available for 3,369 patients. c. Data available for $557 \mathrm{pN}+$ patients. d. Data available for $836 \mathrm{pN}+$ patients. e. Data available for 3,159 patients. f. Data available for 2,293 patients. g. G4 carcinomas are reported in Supporting Information Table S3. Key: ECLNI, extracapsular lymph node involvement. 


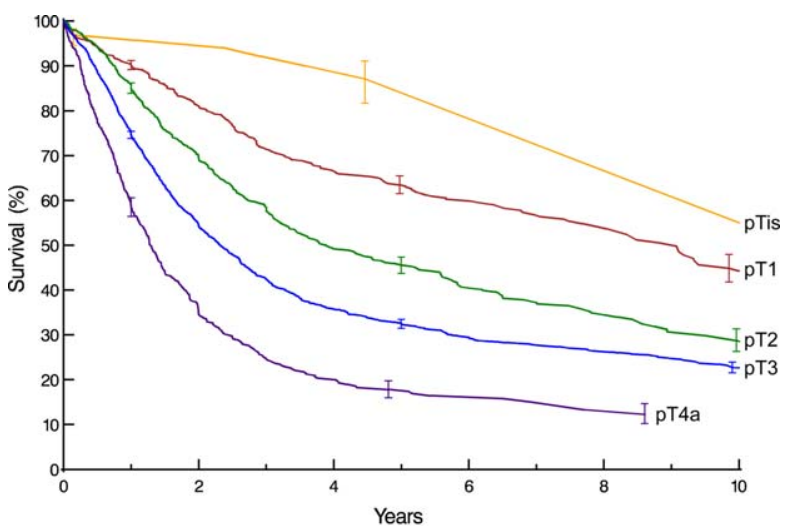

(a)

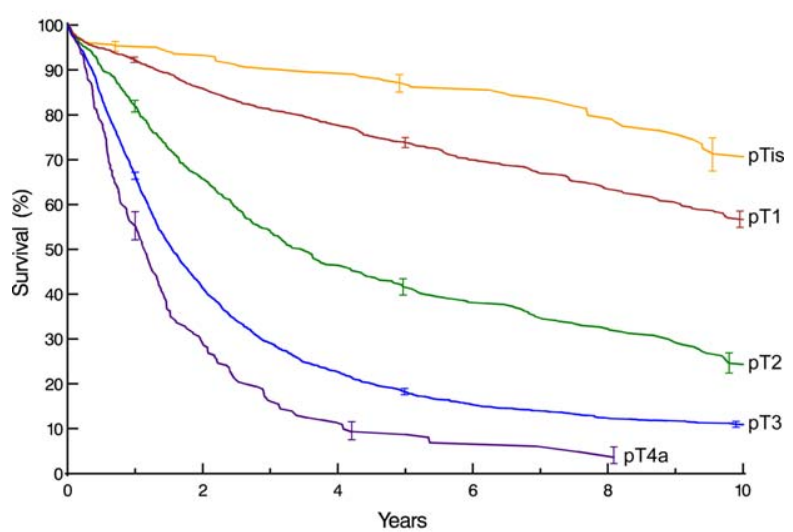

(b)

Fig. 1 Survival stratified by pT category. Kaplan-Meier estimates accompanied by vertical bars representing $68 \%$ confidence limits, equivalent to \pm 1 standard error. A, Squamous cell carcinoma. B, Adenocarcinoma.

squamous cell carcinoma (Fig. 4). Survival decreased with presence of distant metastases (pM; Fig. 5).

\section{Other cancer categories}

Survival decreased with increasing histologic grade for G1-4 cancers (Supporting Information Fig. S5), although it was more distinctive for adenocarcinoma. Survival was higher with a more distal location of cancer within the esophagus for squamous cell carcinoma (Supporting Information Fig. S6).

\section{Other characteristics}

Survival diminished with advancing age (Supporting Information Fig. S7) and was worse for men (Supporting Information Fig. S8).

\section{Treatment}

Survival was similar for patients with squamous cell carcinoma receiving no adjuvant therapy and those receiving adjuvant chemotherapy and chemoradiotherapy (Supporting Information Fig. S9A). It was worse for those receiving adjuvant radiotherapy alone. Survival for patients with adenocarcinoma was best in those not receiving adjuvant therapy, worse but similar for those receiving chemoradiotherapy or chemotherapy, and worst for those receiving radiotherapy (Supporting Information Fig. S9B). Survival was better with increasing number of regional lymph nodes resected for squamous cell carcinoma, but, paradoxically, decreased with increasing regional lymph nodes resected for adenocarcinoma (Supporting Information Fig. S10). Survival decreased dramatically with positive resection margins, and was worst for R2 (Supporting Information Fig. S11).

\section{DISCUSSION}

\section{Principal findings}

Non-risk-adjusted survival for many categories of squamous cell carcinoma had different patterns from adenocarcinoma when treated by esophagectomy or endoscopic therapy without preoperative therapy, particularly for early-stage cancers. This observation suggests the need for separate stage groupings for squamous cell carcinoma and adenocarcinoma, but risk adjustment is necessary to confirm this simple observation for all stage groups. The enlarged data set

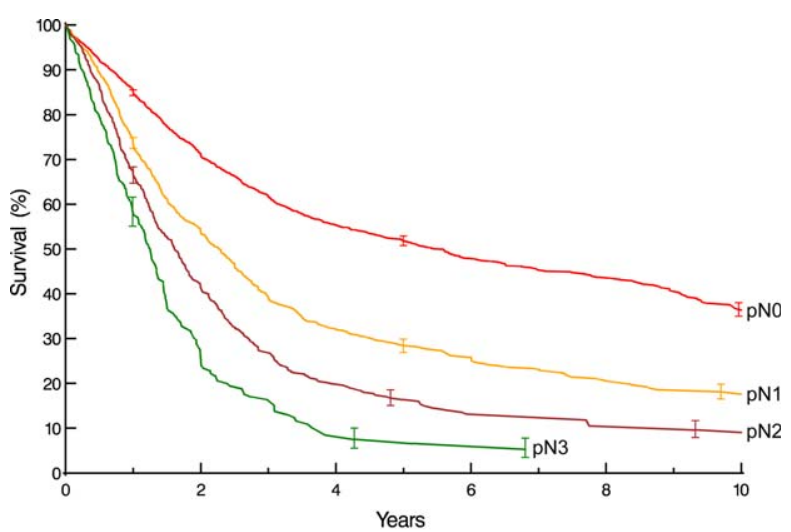

(a)

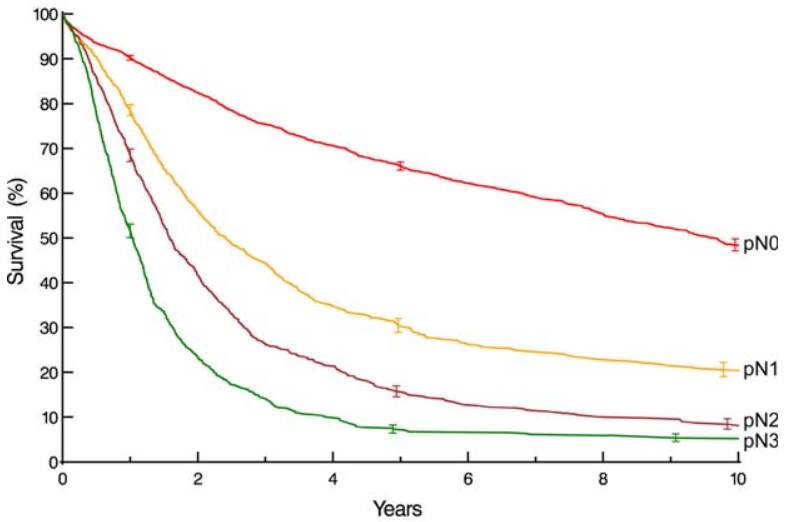

(b)

Fig. 2 Survival stratified by $\mathrm{pN}$ category ( $\mathrm{pN} 1$ is $1-2$ positive nodes, $\mathrm{pN} 2$ is $3-6$ positive nodes, and $\mathrm{pN} 3$ is $\geq 7$ positive nodes). Format is as in Fig. 1. A, Squamous cell carcinoma. B, Adenocarcinoma. 


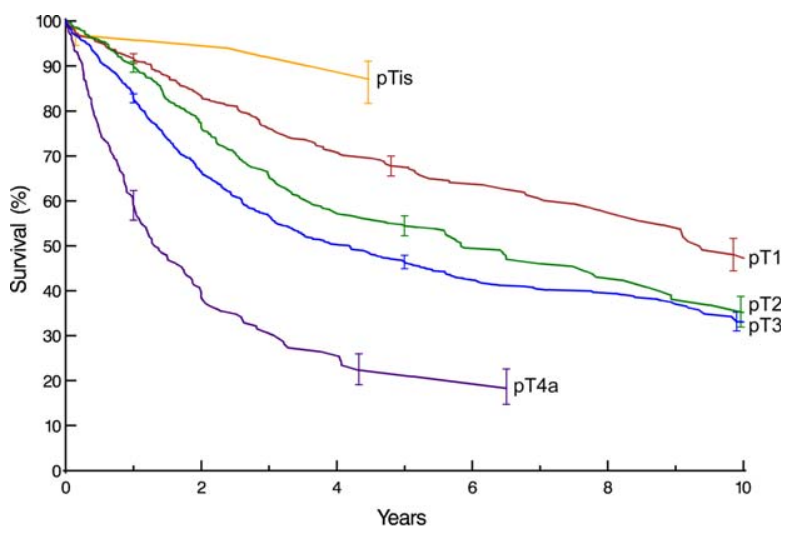

(a)

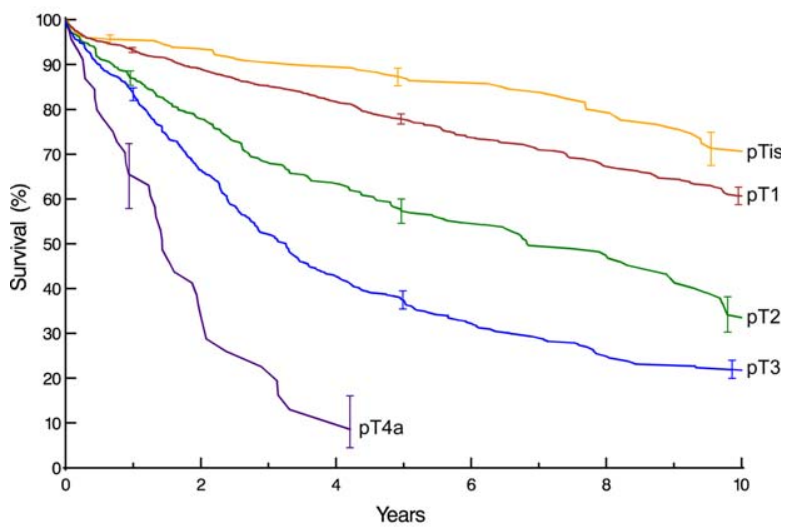

(b)

Fig. 3 Survival stratified by $\mathrm{pT}$ category for $\mathrm{pN} 0$ cancers. Format is as in Fig. 1. A, Squamous cell carcinoma. B, Adenocarcinoma.

demonstrates the need for subcategorization of $\mathrm{T} 1$ cancers and illustrates differences in survival between squamous cell carcinoma and adenocarcinoma for these early cancers.

Sufficient data on use of postoperative adjuvant therapy are now available, and their role needs to be determined in risk-adjusted analysis. The numbers of endoscopic therapies and less invasive esophagectomies in the data set are too small to draw conclusions about their effect on survival.

\section{WECC and data assemblage}

The 7 th edition staging manuals were based on pathologic staging of patients in WECC undergoing esophagectomy alone. 1,2,7 This new WECC effort included collecting more extensive pathologic staging data for three times more patients. The number of patient characteristic variables was greater and the data more complete. It also includes patients who received postoperative adjuvant treatment. The data include patients from six continents, making this a global effort across geography, institutions, patients, cancer categories, and treatments. These data will serve as the basis of the 8th edition cancer staging manuals following risk adjustment for all these variables.

(C) 2016 International Society for Diseases of the Esophagus
Esophagectomy alone is becoming less common as the sole treatment for advanced-stage and preinvasive/ early stage cancers. Therefore, these WECC data may have historical significance, documenting this era of treatment and serving as a reference for future comparison of new treatments with esophagectomy alone.

\section{Patient characteristics}

There were many differences in patient characteristics between histopathologic cell types. These differences were far greater than those seen in patients receiving neoadjuvant therapy. ${ }^{8}$ This likely reflects patient selection for neoadjuvant therapy. These differences necessitate risk adjustment in any future analysis.

\section{Treatment}

The vast majority of patients received esophagectomy, few less invasively. Lymphadenectomy yielded more than 11 regional lymph nodes in $70 \%$ of patients, and more than 20 in $40 \%$. The paradox of better survival of patients with adenocarcinoma who had fewer lymph nodes resected may be explained in part by use of resection approaches that limit extent of lymphadenectomy in patients with early clinically staged cancers. The poor survival of patients with positive resection margins

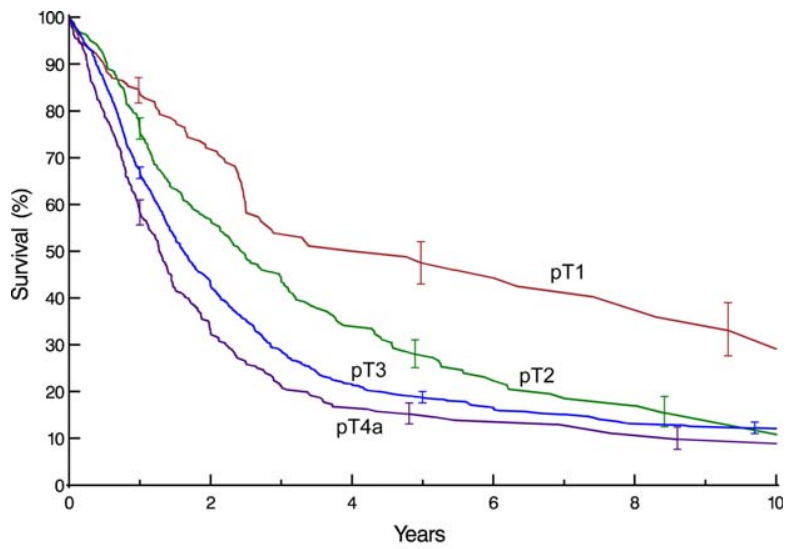

(a)

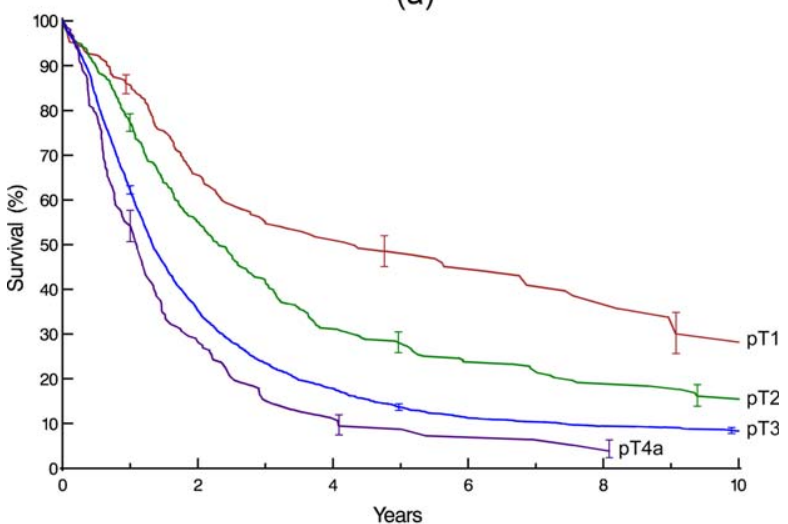

(b)

Fig. 4 Survival stratified by $\mathrm{pT}$ category for $\mathrm{pN}+$ cancers. Format is as in Fig. 1. A, Squamous cell carcinoma. B, Adenocarcinoma. 


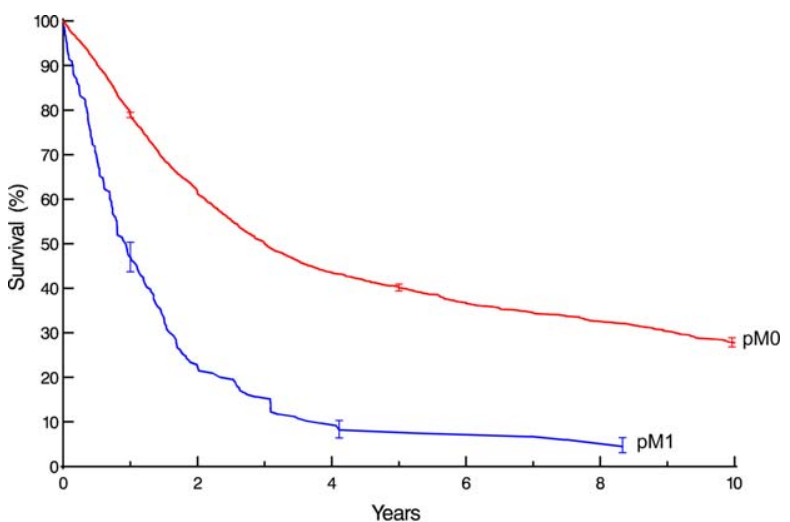

(a)

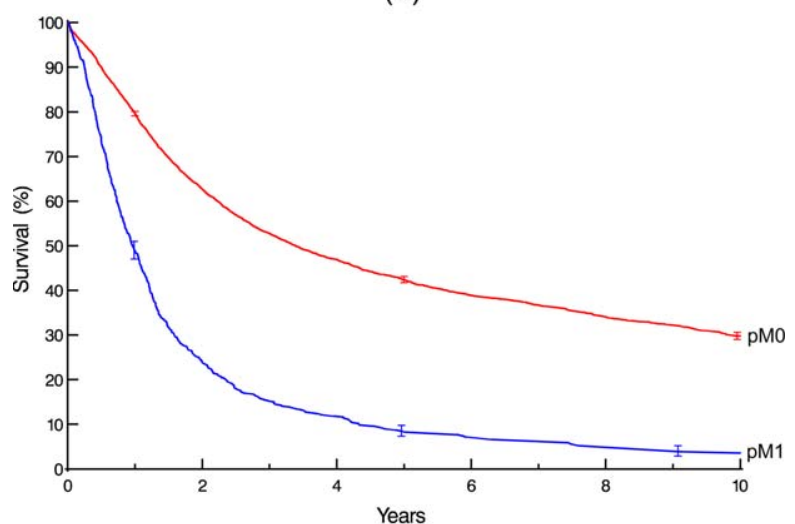

(b)

Fig. 5 Survival stratified by pM category. Format is as in Fig. 1. A, Squamous cell carcinoma. B, Adenocarcinoma.

underscores the importance of complete resection. Postoperative adjuvant therapy was used in twice as many squamous cell carcinoma patients as in adenocarcinoma patients. Despite a concerted effort to enroll centers having extensive experience with endoscopic treatment of superficial esophageal cancers, such patients were underrepresented.

\section{Pathologic cancer categories}

The majority of cancers were advanced. The chances of invasion outside the esophageal wall (pT3-4a) and regional lymph node metastases $(\mathrm{pN}+)$ for adenocarcinoma patients were nearly equal; these chances were approximately $65 \%$ for squamous cell carcinoma patients. Because this was a surgical series, few patients had distant metastases (pM, which includes both nonregional lymph nodes and distant metastases)., Advanced-grade cancer predominated; however, 20\% of patients had G1 cancers. Location was overwhelmingly lower thoracic esophagus in adenocarcinoma patients. There were few patients with adenocarcinoma of the middle thoracic esophagus, and upper thoracic adenocarcinoma was rare. The distribution of location for squamous cell carcinoma was skewed to the middle and lower thoracic esophagus for multiple reasons, including the dominance of surgery in the WECC series. Nevertheless, there are sufficient data to permit analysis of the effect of location on risk-adjusted survival. No patient with cervical esophageal cancer was included in the data.

\section{Non-risk-adjusted survival}

The endpoint for this study was all-cause mortality. ${ }^{3}$ This hard endpoint has been shown to provide a truer reflection of death due to cancer than the softer endpoint of disease-specific mortality. ${ }^{9-11}$

Overall survival was similar for squamous cell carcinoma and adenocarcinoma. This surprising fact reflects important differences in patient and cancer characteristics between these 2 groups. Survival for pTis-1N0M0 adenocarcinomas was much better than for squamous cell carcinoma. Unadjusted survival was more distinctive for adenocarcinoma than squamous cell carcinoma when stratifying $\mathrm{pT}$ by $\mathrm{pN}$. Survival of pM1 patients, although poor, was better than expected, reflecting this atypical pM1 patient population undergoing esophagectomy. Survival was distinctive by histologic grade but less so than in WECC data for the 7th edition, which was less than a third the size of the present WECC data set. The predominance of lower thoracic esophageal adenocarcinoma makes interpreting location effect on survival impossible for adenocarcinoma. Only squamous cell cancer had a sufficient number of patients with both upper and middle esophageal cancers to provide reliable unadjusted survival curves for all locations. Late survival for women is better than for men. Complex interactions require risk adjustment to effectively evaluate the effect of number of lymph nodes resected on survival. Addition of adjuvant therapy was not associated with better non-risk-adjusted survival for patients regardless of cell type. However, interpretation of its effect requires risk adjustment.

\section{Strengths and limitations}

Currently, this is the best attempt at providing worldwide pathologic esophageal cancer staging data. However, patient selection and treatment delivery was not uniform among centers or across continents, and this heterogeneity was reflected in heterogeneous survival. Patients treated in North America, Europe, and Asia predominated. Unlike most registry data, WECC collected more patient characteristics and cancer categories, specific treatment and start dates were known, and follow-up data were available for all. However, not all variables were reported for all patients.

A limitation of this pure data presentation is that it does not account for patient variables that affect allcause mortality; the interplay among TNM, histopathologic cell type, histologic grade, and cancer location, in part due to the unique lymphatic anatomy of the esophagus; and the confounding of treatment effects, temporal factors, etiology, diagnosis, and clinical 
decision-making around the world. This analysis does not account for patients' clinical (pretreatment) cancer characteristics.

The data set reflects temporal changes in resection alone for esophageal cancer. Nevertheless, older data, which may seem a limitation, are crucial for developing pathologic staging of advanced esophageal cancers.

\section{CONCLUSIONS}

This WECC pathologic staging data set is greatly augmented compared with the previous WECC effort. The number of patients and scope of variables collected has been substantially increased. The assemblage of a larger and more complete list of comorbidity data was necessary to allow risk adjustment for the hard endpoint of all-cause mortality.

The need for separate staging of advanced squamous cell carcinoma and adenocarcinoma will be assessed by risk-adjusted analyses of these data. Subcategories of $\mathrm{T} 1$ for both histopathologic cell types is necessary and will be possible. The benefit of postoperative therapy and its effect on pathologic staging awaits risk-adjusted analysis. Data are insufficient to determine if endoscopic or less invasive techniques produce survival similar to esophagectomy in patients with comparable categories.

These data form the basis of pTNM staging for epithelial cancers of the esophagus and esophagogastric junction. However, the role of pure pathologic staging for treatment decision and prognostication for these cancers is waning. ${ }^{4,8}$

\section{Acknowledgments}

The authors thank Brian Kohlbacher for assistance with figure annotation and Tess Parry for manuscript preparation.

\section{Author Contributions}

Conception or design of the experiment(s), or collection and analysis or interpretation of data: all authors. Drafting the manuscript or revising its intellectual content: Rice, Apperson-Hansen, and Blackstone. Approval of the final version of the submitted manuscript: all authors.

\section{References}

1 Edge S B, Byrd D R, Compton C C, Fritz A G, Greene F L, Trotti A, (eds). American Joint Committee on Cancer Staging Manual. New York: Springer-Verlag, 2010.

2 Sobin L H, Gospodarowicz M K, Wittekind C, (eds). TNM Classification of Malignant Tumours. International Union against Cancer. Oxford, England: Wiley-Blackwell, 2009.

3 Rice T, Rusch V, Apperson-Hansen C et al. Worldwide Esophageal Cancer Collaboration. Dis Esophagus 2009; 22: 1-8.

4 Rice T, Apperson-Hansen C, DiPaola L et al. Worldwide Esophageal Cancer Collaboration: clinical staging data. Dis Esophagus 2016 (forthcoming).

5 Goldman A. Eventcharts: visualizing survival and other timedevent data. Am Stat 1992; 46: 13-8.

6 Blackstone E, Naftel D, Turner M. Jr. The decomposition of time-varying hazard into phases, each incorporating a separate stream of concomitant information. J Am Stat Assoc 1986; 81: 615-24.

7 Rice T, Rusch V, Ishwaran H, Blackstone E. Cancer of the esophagus and esophagogastric junction: data-driven staging for the 7th edition of the American Joint Committee on Cancer/International Union Against Cancer staging manuals. Cancer 2010; 116: 3763-73.

8 Rice T, Lerut T E M, Orringer M et al. Worldwide Esophageal Cancer Collaboration: neoadjuvant pathologic staging data. Dis Esophagus 2016; 22: 1-8.

9 van Leeuwen P, Kranse R, Hakulinen T et al. Disease-specific mortality may underestimate the total effect of prostate cancer screening. J Med Screen 2010; 17: 204-10.

10 Black W, Haggstrom D, Welch H. All-cause mortality in randomized trials of cancer screening. J Natl Cancer Inst 2002; 94: $167-73$.

11 Lauer M, Blackstone E, Young J, Topol E. Cause of death in clinical research: time for a reassessment? J Am Coll Cardiol 1999; 34: 618-20.

\section{APPENDIX: Worldwide Esophageal Cancer Collaboration: participating institutions and investigators}

\begin{tabular}{|c|c|c|}
\hline Institution & Location & Investigators \\
\hline Beijing Cancer Hospital, Peking University & Beijing, China & Ken N. Chen \\
\hline Cleveland Clinic & Cleveland, $\mathrm{OH}$; USA & $\begin{array}{l}\text { Thomas W. Rice } \\
\text { Eugene H. Blackstone }\end{array}$ \\
\hline Case Western Reserve University & Cleveland, OH; USA & Carolyn Apperson-Hansen \\
\hline Erasmus Medical Center & Rotterdam, The Netherlands & $\begin{array}{l}\text { Bas P.L. Wijnhoven } \\
\text { Jan van Lanschot } \\
\text { Sjoerd Lagarde }\end{array}$ \\
\hline Fourth Hospital of Hebei Medical University & Shijiazhuang, Hebei; China & Jun-Feng Liu \\
\hline Fox Chase Cancer Center & Philadelphia, PA; USA & $\begin{array}{l}\text { Walter J. Scott } \\
\text { Donna Edmondson }\end{array}$ \\
\hline Groote Schuur Hospital, University of Cape Town & Cape Town, South Africa & Riette Burger \\
\hline Guy's \& St Thomas' Hospitals & London, UK & $\begin{array}{l}\text { Andrew R. Davies } \\
\text { Janine Zylstra }\end{array}$ \\
\hline Helsinki University Hospital & Helsinki, Finland & $\begin{array}{l}\text { Jari V. Räsänen } \\
\text { Jarmo A. Salo } \\
\text { Yvonne Sundstrom }\end{array}$ \\
\hline Hospital Universitario del Mar & Barcelona, Spain & Manuel Pera \\
\hline Hôpital Nord & Marseille, France & Xavier B. D'Journo \\
\hline
\end{tabular}


Appendix (Continued)

\begin{tabular}{|c|c|c|}
\hline Institution & Location & Investigators \\
\hline Indiana University Medical Center & Indianapolis, IN; USA & Kenneth A. Kesler \\
\hline University of Texas MD Anderson Hospital & Houston, TX; USA & $\begin{array}{l}\text { Wayne L. Hofstetter } \\
\text { Arlene Correa } \\
\text { Stephen G. Swisher }\end{array}$ \\
\hline Mayo Clinic & Rochester, MN; USA & Mark S. Allen \\
\hline Medical University of South Carolina & Charleston, SC; USA & Chad E. Denlinger \\
\hline Memorial Sloan-Kettering Cancer Center & New York, NY; USA & Valerie W. Rusch \\
\hline University of Queensland, Princess Alexandra Hospital & Brisbane, Australia & $\begin{array}{l}\text { B. Mark Smithers } \\
\text { David Gotley } \\
\text { Andrew Barbour } \\
\text { Iain Thomson }\end{array}$ \\
\hline University of Newcastle upon Tyne & Newcastle upon Tyne, UK & $\begin{array}{l}\text { S. Michael Griffin } \\
\text { Jon Shenfine }\end{array}$ \\
\hline Oregon Health \& Science University & Portland, OR; USA & $\begin{array}{l}\text { Paul H. Schipper } \\
\text { John G. Hunter }\end{array}$ \\
\hline Royal Marsden NHS Foundation Trust & London, UK & William H. Allum \\
\hline Shanghai Chest Hospital & Shanghai, China & Wentao (Vincent) Fang \\
\hline Toronto General Hospital & Toronto, ON; Canada & Gail E. Darling \\
\hline University Zeikenhuizen Leuven & Leuven, Belgium & $\begin{array}{l}\text { Tony E.M.R. Lerut } \\
\text { Phillipe R. Nafteux }\end{array}$ \\
\hline University Medical Center Utrecht & Utrecht, The Netherlands & Richard van Hillegersberg \\
\hline University of Alabama at Birmingham & Birmingham, AL; USA & Robert J. Cerfolio \\
\hline Hospital de Clinicas, University of Buenos Aires & Buenos Aires, Argentina & $\begin{array}{l}\text { Luis Durand } \\
\text { Roberto De Antón }\end{array}$ \\
\hline The University of Chicago, Department of Surgery & Chicago, IL; USA & Mark K. Ferguson \\
\hline $\begin{array}{l}\text { University of Hong Kong Medical Center, } \\
\text { Queen Mary Hospital }\end{array}$ & Hong Kong, China & Simon Law \\
\hline University of Michigan & Ann Arbor, MI; USA & $\begin{array}{l}\text { Mark B. Orringer } \\
\text { Becky L. Marshall }\end{array}$ \\
\hline University of Montreal & Montreal, Quebec; Canada & $\begin{array}{l}\text { André Duranceau } \\
\text { Susan Howson }\end{array}$ \\
\hline University of Pittsburgh Medical Center & Pittsburgh, PA; USA & $\begin{array}{l}\text { James D. Luketich } \\
\text { Arjun Pennathur } \\
\text { Kathy Lovas }\end{array}$ \\
\hline University of Rochester & Rochester, NY; USA & Thomas J. Watson \\
\hline University of São Paulo & São Paulo, Brazil & Ivan Cecconello \\
\hline West China Hospital of Sichuan University & Chengdu, Sichuan; China & Long-Qi Chen \\
\hline
\end{tabular}

West China Hospital of Sichuan University

São Paulo, Brazil

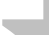

$\sqrt{2}$

$\sqrt{-2 \cdot(2)}$

\section{SUPPORTING INFORMATION}

Additional Supporting Information may be found in the online version of this article at the publisher's web-site:

Fig. S1: Survival after pathologic staging of esophageal cancer patients. Vertical bars on KaplanMeier estimates represent $68 \%$ confidence limits, equivalent to \pm 1 standard error.

Fig. S2: Instantaneous risk of death (hazard function). Dashed lines represent $68 \%$ confidence limits.

Fig. S3: Survival stratified by subcategories of pT1. Format is as in Supporting Information Fig. S1. pTis and pT2 are included for reference. A, Squamous cell carcinoma. B, Adenocarcinoma.

Fig. S4: Survival stratified by number of cancerpositive locoregional lymph nodes. Format is as in Supporting Information Fig. S1. A, Squamous cell carcinoma. B, Adenocarcinoma.

Fig. S5: Survival stratified by histologic grade (cG1, well differentiated; cG2, moderately differentiated; cG3, poorly differentiated; cG4, undifferentiated). Format is as in Supporting Information Fig. S1. A, Squamous cell carcinoma. B, Adenocarcinoma.

Fig. S6: Survival stratified by upper extent of cancer in the esophagus (location: upper, middle, lower). Format is as in Supporting Information Fig. S1. A, Squamous cell carcinoma. B, Adenocarcinoma.

Fig. S7: Survival stratified by patient age $(<60$, $60-70,>70$ years). Format is as in Supporting Information Fig. S1. A, Squamous cell carcinoma. B, Adenocarcinoma.

Fig. S8: Survival stratified by sex. Format is as in Supporting Information Fig. S1. A, Squamous cell carcinoma. B, Adenocarcinoma.

Fig. S9: Survival stratified by adjuvant therapy. Format is as in Supporting Information Fig. S1. 
Key: $C R T$, chemoradiotherapy. A, Squamous cell carcinoma. B, Adenocarcinoma.

Fig. S10: Survival stratified by number of regional lymph nodes resected. Format is as in Supporting Information Fig. S1. A, Squamous cell carcinoma. B, Adenocarcinoma.

Fig. S11: Survival stratified by resection margin (R0, cancer-free; R1, microscopic; R2, macroscopic). Format is as in Supporting Information Fig. S1. A, Squamous cell carcinoma. B, Adenocarcinoma.

Table S1. Characteristics of patients receiving resection or ablation without preoperative therapy
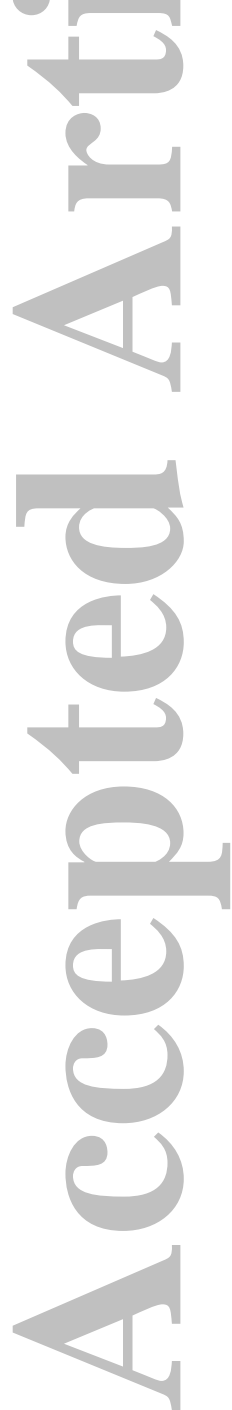

for adenosquamous and undifferentiated carcinoma of the esophagus.

Table S2. Treatment received by patients for adenosquamous and undifferentiated carcinoma of the esophagus.

Table S3. Pathologic cancer categories of patients receiving resection or ablation without preoperative therapy for adenosquamous and undifferentiated carcinoma of the esophagus. 